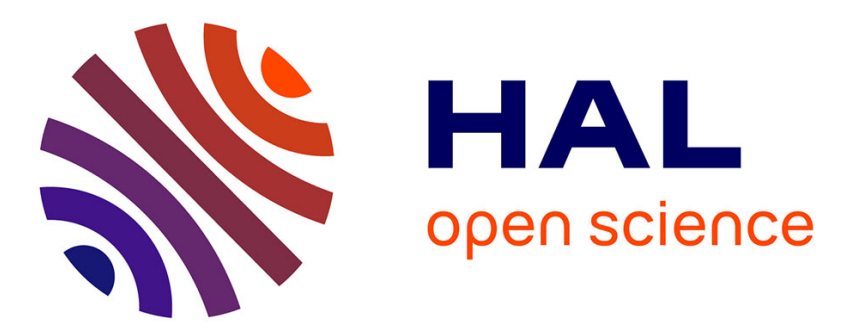

\title{
Model for coherence transfer in a backward optical parametric oscillator
}

\author{
Carlos Montes, Pierre Aschieri, Antonio Picozzi
}

\section{To cite this version:}

Carlos Montes, Pierre Aschieri, Antonio Picozzi. Model for coherence transfer in a backward optical parametric oscillator. 22nd Congress of the International Commission for Optics: Light for the Development of the World, Aug 2011, France. pp.801136, 10.1117/12.901902 . hal-00647509

\section{HAL Id: hal-00647509 \\ https://hal.science/hal-00647509}

Submitted on 2 Dec 2011

HAL is a multi-disciplinary open access archive for the deposit and dissemination of scientific research documents, whether they are published or not. The documents may come from teaching and research institutions in France or abroad, or from public or private research centers.
L'archive ouverte pluridisciplinaire HAL, est destinée au dépôt et à la diffusion de documents scientifiques de niveau recherche, publiés ou non, émanant des établissements d'enseignement et de recherche français ou étrangers, des laboratoires publics ou privés. 


\title{
Model for Coherence Transfer in a Backward Optical Parametric Oscillator
}

\author{
Carlos Montes ${ }^{1, \star}$, Pierre Aschieri ${ }^{1}$, and Antonio Picozzi ${ }^{2}$ \\ 1. LPMC-CNRS, Université de Nice-Sophia Antipolis, Parc Valrose, \\ F-06108 Nice, France \\ 2. Institut Carnot de Bourgogne, F-21078 Dijon Cedex, France
}

\begin{abstract}
The mirrorless backward optical parametric oscillator (BOPO), where the signal and idler waves are propagating in opposite directions, will establish a distributed feedback mechanism and thus optical parametric oscillation without the need to apply mirrors or external feedback to the cavity. It has been recently demonstrated experimentally by exploiting the periodic poling technique in second-order nonlinear crystals, that the sub-micrometer structured medium achieves an efficient quasi-phase-matching of the three wave interaction in the backward configuration. A remarkable property of such BOPO is the high degree of coherence of the backward wave component, whose spectrum may be several order of magnitudes narrower than that of the pump, due to the convectioninduced phase-locking mechanism. Experimentally and numerically proved the transfer of coherent phase modulations from the pump wave to the parametrically down-converted waves, we show here that this is also possible for a broad bandwidth spectrally incoherent pump. In order to accurately describe the nonlinear counter-propagation dynamics of the three dispersive waves, we have developed for the first time to our knowledge a new numerical scheme which combines the method of the trajectories usually employed to solve the three-wave interaction and the intraband group velocity dispersion effect is performed in the spectral domain with the help of the Fast Fourier Transform (FFT) technique. The model accurately conserves the number of photons and the Manley-Rowe invariants. This allowed us to predict various configurations of MOPOs in which, thanks to the convection-induced phase-locking mechanism, a highly coherent backward wave is spontaneously generated from a highly incoherent pump wave.
\end{abstract}

Keywords: nonlinear coherence transfer, backward mirrorless OPO, counterpropagating three wave interactions

$\star$ carlos.montes@unice.fr

\section{Introduction}

It has been theoretically predicted [1] that counter-propagating parametric interactions, where the signal and idler waves are propagating in opposite directions, will establish a distributed feedback mechanism and thus optical parametric oscillation without the need to apply mirrors or external feedback to the cavity. Such a device, the mirrorless optical parametric oscillator (MOPO), has been recently realized by employing engineered second-order nonlinear crystals, namely, periodically poled $\mathrm{KTiOPO}_{4}$ (PPKTP) with the periodicity of the structure of $800 \mathrm{~nm}$ [2]. One of the remarkable properties of the MOPO was a strong asymmetry in the spectral bandwidth of the signal and idler pulses: the signal spectral bandwidth was of the same order as the pump, whereas the counterpropagating idler wave was characterized by a spectral bandwidth about two orders of magnitude narrower with respect to those of the signal and pump waves [3]-[5]. It has been recently experimentally and numerically investigated that this effect occurs when the pump waves exhibits coherent phase modulations: the phase modulations of the pump are coherently tranferred to the co-propagating parametric wave, while the 
counter-propagating wave retains a narrow bandwidth and high coherence [6]. Our aim in this work is to prove that this effect also works when the pump is strongly incoherent characterized by a broad bandwidth stochastic spectrum.

The mechanism underlying such a coherence transfer process is based on a convection-induced phase-locking mechanism [7]-[10]. In substance, the random phase modulations of the pump are transferred to the co-propagating wave, which "absorbs" the incoherence of the pump wave and thus allows the counter-propagating wave to evolve toward a highly coherent state. For this purpose we have developed a new numerical scheme for the integration of the counter-propagating three-wave interaction in the presence of group velocity dispersion. The numerical scheme combines the method of the trajectories usually employed to solve the nonlinear three-wave interaction and the intraband group velocity dispersion effect is performed in the spectral domain with the help of the Fast Fourier Transform (FFT) technique. To our knowledge this combined numerical method has been rendered stable for the first time. We use it to provide accurate numerical simulations of the referred experimental results on the dynamics of broad bandwidth pumped MOPOs.

The mirrorless optical parametric oscillator (MOPO) relies on counter-propagating three wave mixing (TWM) for establishing a distributed positive feedback for sustained oscillation above threshold [11]. It is a general property of TWM that the total interaction phase is fixed. In the case of a down-conversion process, the phases $\phi_{j}$ (where $j=p, s, i$ ) satisfy the relation:

$$
\phi_{p}-\phi_{s}-\phi_{i}=\pi / 2 \text {. }
$$

Here we demonstrate that the maximized convective wave separation of the counter-propagating TWM is responsible for the unusual spectral and coherence properties of the generated signal and idler waves. The physical mechanism ensures that, for a broadband incoherent pump, the frequency bandwidth of the backward-propagating wave, either the idler for the already realized experience or the signal for the here proposed experience, will be substantially narrower and given by the backward wave coherence time. As a consequence of the phase relation in Eq.(1), the phase modulation of the pump is then effectively transferred to the co-propagating parametric wave. The major difficulty in achieving mirrorless parametric oscillation is associated with satisfying the momentum conservation condition, which in scalar form reads $k_{p}=k_{s}-k_{i}$ for the backward idler or $k_{p}=k_{i}-k_{s}$ for the backward signal. In homogeneous dielectrics, the phase matching condition can possibly be satisfied only for large signal-idler detunings. However, due to the very different diffraction properties of the near-infrared signal and the far-infrared idler, achieving oscillation in homogeneous dielectrics is problematic. By employing ferroelectric nonlinear crystals structured with sub-micrometer periodicity, $\Lambda_{\mathrm{QPM}}=2 \pi /\left(k_{p}-k_{s}+k_{i}\right) \simeq 800 \mathrm{~nm}$, quasi-phase matching (QPM) [12] has been utilized to achieve MOPO with counter-propagating idler $k_{i}$ in the mid-infrared spectral range [2] [6]. We show in Fig.1 the different OPO resonant wave vector configurations: three copropagating waves, backward idler wave, backward signal wave, and backward signal and idler waves. The quasi-phase matching periodicity $\Lambda_{\mathrm{QPM}}=2 \pi / \mathrm{K}_{\mathrm{G}}$ runs from several microns for the first to a fourth of micron for the last.

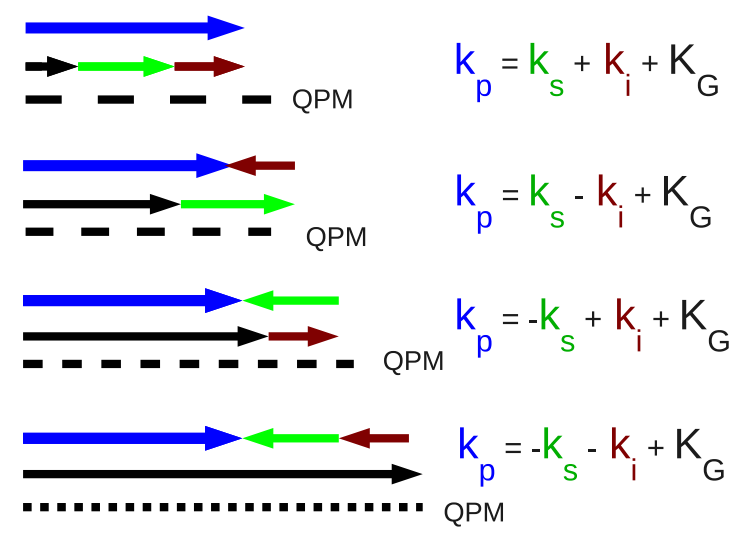

Fig. 1: Resonant wave vector configurations for OPOs 


\section{Nonlinear model: Coherence transfer}

The three-wave interaction is known to model the evolution of the slowly-varying envelopes, $\mathbf{A}_{\mathbf{j}}(\mathrm{j}=\mathrm{p}, \mathrm{s}, \mathrm{i})$, of the three coupled pump, signal and idler waves

$$
\begin{aligned}
\left(\partial_{t}+\mathrm{v}_{\mathrm{p}} \partial_{x}+\gamma_{p}+i \beta_{p} \partial_{t t}\right) \mathbf{A}_{\mathbf{p}} & =-\sigma_{p} \mathbf{A}_{\mathbf{s}} \mathbf{A}_{\mathbf{i}} \\
\left(\partial_{t}+\mathrm{v}_{\mathrm{s}} \partial_{x}+\gamma_{s}+i \beta_{s} \partial_{t t}\right) \mathbf{A}_{\mathbf{s}} & =\sigma_{s} \mathbf{A}_{\mathbf{p}} \mathbf{A}_{\mathbf{i}}^{*} \\
\left(\partial_{t}-\mathrm{v}_{\mathrm{i}} \partial_{x}+\gamma_{p}+i \beta_{i} \partial_{t t}\right) \mathbf{A}_{\mathbf{i}} & =\sigma_{i} \mathbf{A}_{\mathbf{p}} \mathbf{A}_{\mathbf{s}}^{*}
\end{aligned}
$$

where $\sigma_{j}=2 \pi \mathrm{d}_{\mathrm{eff}} \mathrm{v}_{\mathrm{j}} / \lambda_{j} n_{j}$ are the nonlinear coupling coefficients, $\mathrm{d}_{\mathrm{eff}}$ the efficient quadratic susceptibility, and $\gamma_{j}$ and $\beta_{j}$ the damping and dispersion coefficients. The idler $\mathbf{A}_{\mathbf{i}}$ wave is counter-propagating with respect to the pump. The quasi-phase-matching conditions in the backward configuration read

$$
\omega_{p}=\omega_{s}+\omega_{i} \quad \text { and } \quad k_{p}-k_{s}+k_{i}=K_{G}=2 \pi m / \Lambda_{\mathrm{QPM}},
$$

where $K_{G}$ is the quasi phase-matching (QPM) grating pitch vector, $m$ is the order of the interaction and $\Lambda_{\mathrm{QPM}}=\left[n_{p} / \lambda_{p}-\right.$ $\left.n_{s} / \lambda_{s}+n_{i} / \lambda_{i}\right]^{-1}$ is the modulation period of the quadratic nonlinear coefficient.

The plane wave monochromatic model [11] satisfactorily estimates the order of magnitude of the MOPO threshold for the counter-propagating interaction, namely $I_{\mathrm{pth}}=c \varepsilon_{0} n_{p} n_{s} n_{i} \lambda_{s} \lambda_{i} / 2 \mathrm{~L}^{2} \mathrm{~d}_{\mathrm{eff}}^{2}$. Therefore, considering as in the experimental studies [2] [6] a crystal length of $\mathrm{L}=0.65 \mathrm{~cm}$, one obtains $I_{\mathrm{pth}}=1.12 \mathrm{GW} / \mathrm{cm}^{2}$. Note that such a MOPOs threshold may increase substantially in the presence of an incoherent pump.

We have investigated theoretically the process of phase modulation transfer in MOPO by numerically solving the coupled wave equations and employing different models for the pump pulse, including chirped pulses and pulses containing random phase modulations. The numerical results support very well the experimental results. Note that we have already considered the convection-induced phase-locking mechanism, and the associated process of coherent wave generation from incoherent excitation, in the usual forward configuration of singly resonant (integrated) optical parametric oscillators (SIOPO) [8] - [10]. In particular, it was numerically proved that the waveguide geometry considerably increases the conversion efficiency of the nonlinear device, which thus permits to make use of a low-power cw-pumping [13] - [17]. However, the conventional forward resonant OPOs impose severe constraints on the resonant phase-matching conditions: one needs to match the group velocity of the pump wave with one of the daughter waves, while still preserving a large group velocity difference (i.e. large convection) with the other daughter wave [9] [10]. We underline that this last condition is automatically satisfied in the backward MOPO, since the counter-propagating waves ensure a maximum convection, so that we just need to match the group velocity of the pump with the other wave.

\subsection{Convection-induced phase-locking mechanism}

The coherence properties of the parametric three-wave interaction driven from an incoherent pump has been the subject of an analytical study where the autocorrelation functions are mathematically evaluated in the presence of dispersion [7] [8] [18], and the convection-induced phase-locking mechanism has been proposed for the forward OPOs configurations [9] [10]. Let us present here some simple analytical arguments enlighting this mechanism from equations (2) for the backward idler configuration.

Let us consider the dispersionless case $\left(\beta_{j}=0\right), \sigma_{s}=\sigma_{i}=\sigma_{p} / 2=\sigma$, and the linear undepleted pump limit with $\gamma_{p}=0$. The incoherent pump may be modeled as a stationary single-variable stochastic function $\mathbf{A}_{\mathbf{p}}(\mathbf{z})$ with the following autocorrelation function

$$
\left\langle\mathbf{A}_{\mathbf{p}}\left(\mathrm{z}-\mathrm{z}^{\prime}\right) \frac{\mathbf{A}_{\mathbf{p}}{ }^{*}\left(\mathbf{z}^{\prime}\right)}{\left|\mathbf{A}_{\mathbf{p}}(0)\right|^{2}}\right\rangle=\exp \left(-\frac{|\mathbf{z}|}{\lambda_{c}}\right),
$$

$\lambda_{c}=\mathrm{v}_{\mathrm{p}} \tau_{c}$ being the correlation length of the pump in its own traveling frame,

$$
\mathrm{z}=\mathrm{x}-\mathrm{v}_{\mathrm{p}} \mathrm{t}
$$

The correlation time is $\tau_{\mathrm{c}} \simeq 1 / \pi \Delta v_{\mathrm{p}}$, where $\Delta v_{p}$ is the (broad)-bandwidth of the incoherent pump spectrum. The role of convection in the coherence of the daughter waves $\mathbf{A}_{\mathbf{s}}$ and $\mathbf{A}_{\mathbf{i}}$ may be analyzed by integrating the second equation (2) along the characteristic of the signal wave. Then, the third equation (2) yields

$$
\mathrm{DA}_{\mathbf{i}}=\sigma^{2} \int_{0}^{\mathrm{t}} \mathrm{e}^{-\gamma_{\mathbf{s}}\left(\mathrm{t}-\mathrm{t}^{\prime}\right)} \mathbf{A}_{\mathbf{p}}(\mathrm{z}) \mathbf{A}_{\mathbf{p}}{ }^{*}\left(\mathrm{z}^{\prime}\right) \mathbf{A}_{\mathbf{i}}\left(\mathrm{x}^{\prime}, \mathrm{t}^{\prime}\right) \mathrm{dt^{ \prime }}
$$


where

$$
\begin{gathered}
\mathrm{D}=\partial / \partial \mathrm{t}-\mathrm{v}_{\mathrm{i}} \partial / \partial \mathrm{x}+\gamma_{\mathrm{i}} \\
\mathrm{z}^{\prime}=\mathrm{z}-\left(\mathrm{v}_{\mathrm{s}}-\mathrm{v}_{\mathrm{p}}\right)\left(\mathrm{t}-\mathrm{t}^{\prime}\right) ; \mathrm{x}^{\prime}=\mathrm{x}-\mathrm{v}_{\mathrm{s}}\left(\mathrm{t}-\mathrm{t}^{\prime}\right)
\end{gathered}
$$

If $\mathrm{v}_{\mathrm{s}}=\mathrm{v}_{\mathrm{p}}$ we have $\mathrm{z}^{\prime}=\mathrm{z}$ and we can extract the pump amplitude from the integral, i.e. $\mathbf{A}_{\mathbf{p}}(\mathrm{z}) \mathbf{A}_{\mathbf{p}}{ }^{*}\left(\mathrm{z}^{\prime}\right)=\left|\mathbf{A}_{\mathbf{p}}(\mathrm{z})\right|^{2}$, which reveals that the idler evolution is independent of the pump phase fluctuations $\Phi_{p}(\mathrm{z})$. This means that the rapid random phase fluctuations of the pump do not affect the idler, which undergoes slow phase variations and thus evolves towards a highly coherent state during its parametric amplification.

Let us now consider the evolution of the signal wave from the second equation (2):

$$
\mathbf{A}_{\mathbf{s}}(\mathrm{x}, \mathrm{t})=\sigma \int_{0}^{\mathrm{t}} \mathrm{e}^{-\gamma_{\mathbf{s}}\left(\mathrm{t}-\mathrm{t}^{\prime}\right)} \mathbf{A}_{\mathbf{p}}\left(\mathrm{z}^{\prime}\right) \mathbf{A}_{\mathbf{i}}{ }^{*}\left(\mathrm{x}^{\prime}, \mathrm{t}^{\prime}\right) \mathrm{dt}^{\prime}
$$

When $\mathrm{v}_{\mathrm{s}}=\mathrm{v}_{\mathrm{p}}$ we have $\mathrm{z}^{\prime}=\mathrm{z}$ and $\mathbf{A}_{\mathbf{p}}\left(\mathrm{z}^{\prime}\right)$ becomes independent of $\mathrm{t}^{\prime}$, which leads to a signal amplitude $\mathbf{A}_{\mathbf{s}}$ that is simply proportional to the pump amplitude $\mathbf{A}_{\mathbf{p}}$ i.e., the signal field absorbs the noise of the co-moving pump field. Note that this pump-signal phase-locking mechanism does not require an exact matching of the group velocities $\mathrm{v}_{\mathrm{s}}=\mathrm{v}_{\mathrm{p}}$. It is indeed sufficient that

$$
\left|\mathrm{v}_{\mathrm{s}}-\mathrm{v}_{\mathrm{p}}\right| \ll \lambda_{\mathrm{c}} \gamma_{\mathrm{s}}=\mathrm{v}_{\mathrm{p}} \tau_{\mathrm{c}} \gamma_{\mathrm{s}},
$$

to remove the pump field from the integral so that the idler field follows the rapid pump phase fluctuations.

\subsection{Highly incoherent pump pulse}

In order to test the new numerical model governing the dispersive three-wave interaction, we have studied the backward MOPO pumped by a highly incoherent pump pulse. The numerical scheme combines the method of the trajectories that proved efficient in solving the large convections involved in the counterpropagating configuration of the three wave interaction through a Runge-Kutta algorithm of 4th order [19, 20], together with the spectral method of fast Fourier transform (FFT) that integrates the second-order temporal derivatives of Eqs.(2) in the Fourier frequency domain. To our knowledge, this is the first time that these two fundamentally different numerical schemes are combined to integrate the counterpropagating dispersive TWI. The scheme of the model is shown in figure 2. The highly incoherent pump pulse is injected at the entry of the MOPO box (of lenght L) where the nonlinear three wave interaction takes place starting from signal and idler spontaneous noises. For solving the nonlinear time evolution in Eqs.(2) we use the well known numerical model which follows the trajectories and where the integration is done through the Runge-Kutta algorithm. A larger box $(\mathrm{M}=\mathrm{L}+2 \mathrm{~d})$ including the first one, discretized in $2^{\mathrm{N}}$ ( $\mathrm{N}=16$ to 18) points, is used to perform the FFT for evaluating the dispersion terms in Eqs.(2). Smoothed decreasing curves to zero join the input and output wave amplitudes to the ends of this box for correctly performing the FFT.

Let us briefly comment some interesting experiments where the process of incoherent parametric excitation has been investigated. In Refs.[21]-[23] the authors experimentally demonstrate that a coherent amplification may be achieved for a single signal wave through its coupling with two distinct pump beams that are not correlated with each other. In other terms, for specific phase-matching conditions, a single signal wave may be phase matched to a couple of pump waves and to the corresponding set of idler waves, so that the signal mode may be efficiently amplified by taking advantage of the two distinct uncorrelated pump beams simultaneously. Moreover, this process of cumulative pump action has also been observed in the spatial domain owing to conical optical beams by exploiting their specific phase-matching conditions [24] [25]. Although these experiments corroborate the results of the convection-induced phase-locking mechanism, it would be of great interest to observe in a straightforward way the predicted phenomenon of incoherent excitation of a coherent signal in a MOPO. Let us also mention that the convection-induced phase-locking mechanism has been also recently predicted to occur in an optical fiber system by exploiting the peculiar dispersion properties of photonic crystal fibers [26].

In the numerical simulations we consider an incoherent pump pulse of spectral bandwidth $\Delta v_{p}=15 \mathrm{THz}$, for the experimentally accessible wavelengths: $\lambda_{\mathrm{p}}=861.67 \mathrm{~nm}, \lambda_{\mathrm{s}}=1217.94 \mathrm{~nm}$, and $\lambda_{\mathrm{i}}=2945.73 \mathrm{~nm}$. This point of operation is plotted on the group velocity dispersion curves reported in figure 3 . The crystal length of $\mathrm{L}=0.65 \mathrm{~cm}$ in the MOPO provides a photon flight time in the cavity $t_{r}=\mathrm{L} / \mathrm{v}_{\mathrm{p}}=41 \mathrm{ps}$ and a FSR $=\mathrm{v}_{\mathrm{p}} /(2 \mathrm{~L})=12 \mathrm{GHz}$. This configuration corresponds to $\mathrm{L}=14 \Lambda_{0}$, where $\Lambda_{0}=\mathrm{v}_{\mathrm{p}} \tau_{0}=0.46 \mathrm{~mm}$ is the nonlinear characteristic length.

The corresponding KTP refractive index, group velocity and dispersion coefficient for the pump are $\mathrm{n}_{\mathrm{p}}=1.84044, \mathrm{v}_{\mathrm{p}} / \mathrm{c}=0.526359$, $\beta_{\mathrm{p}} / \mathrm{v}_{\mathrm{p}}=0.254223 \mathrm{ps}^{2} / \mathrm{m}$; respectively for the signal are $\mathrm{n}_{\mathrm{s}}=1.824484, \mathrm{v}_{\mathrm{s}} / \mathrm{c}=0.537107, \beta_{\mathrm{s}} / \mathrm{v}_{\mathrm{s}}=0.142531 \mathrm{ps}^{2} / \mathrm{m}$; and 


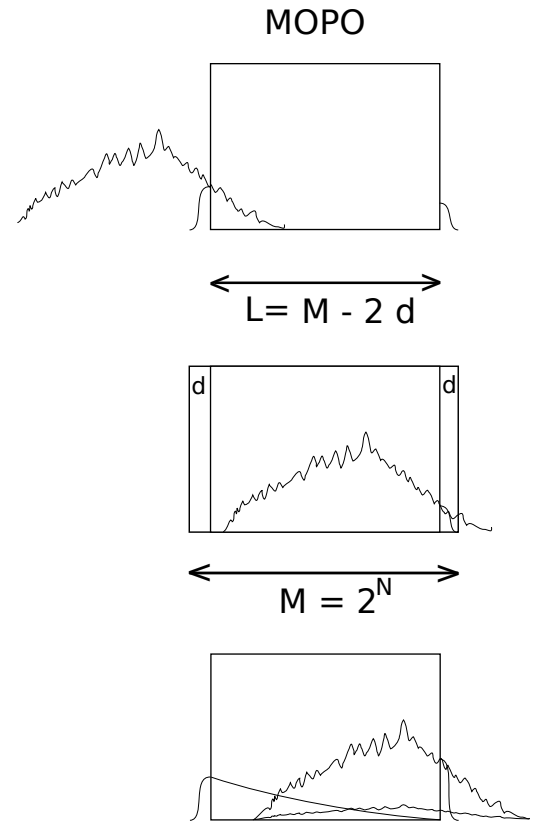

Fig. 2: Scheme for the Runge-Kutta-FFT numerical model in the backward mirrorless OPO.

respectively for the idler are $n_{i}=1.784471, v_{i} / c=0.537301, \beta_{i} / v_{i}=-0.328753 \mathrm{ps}^{2} / m$. The group velocity difference between the pump and the co-propagating signal is $\left|\mathrm{v}_{\mathrm{p}}-\mathrm{v}_{\mathrm{s}}\right| / \mathrm{v}_{\mathrm{s}} \simeq 0.02$.

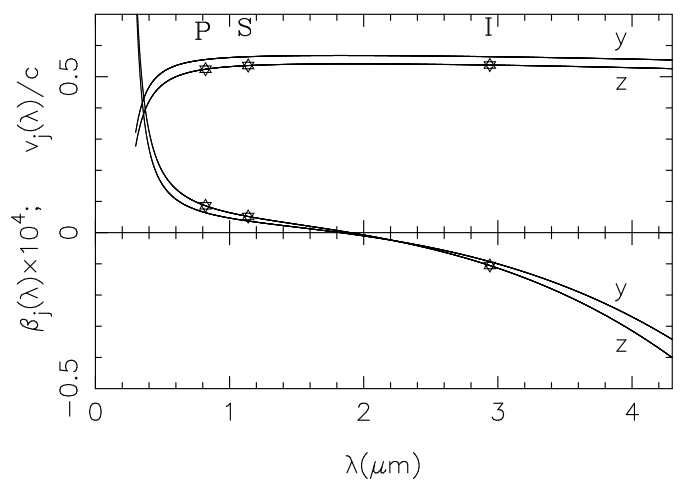

Fig. 3: Group velocity dispersion for $\mathrm{KTiOPO}_{4}$ at $20^{\circ} \mathrm{C}$ (MOPO-1 of type I).

The incoherent pump pulse is characterized by an exponential-shaped correlation function $<\mathbf{A}_{\mathbf{p}}\left(x=0, t^{\prime}+t\right) \mathbf{A}_{\mathbf{p}}{ }^{*}(x=0, t)>=\mathbf{A}_{\mathbf{p}}{ }^{2} \exp \left(-|t| / \tau_{c}\right)$, where $\tau_{c}=1 / \pi \Delta v_{p}$ is the correlation time and $\Delta v_{p}$ the bandwidth of the pump spectrum. The results are shown in Figs.4(a)-(c) and Figs.5(a)-(c) for a FWHM pump pulse duration of 100 ps and a pump intensity of $I_{p}=4 \mathrm{GW} / \mathrm{cm}^{2}$.

In Fig.4(a)-(c) we see that the MOPO starts oscillating after an initial delay of $120 \mathrm{ps}$ and then the parametric waves increase up to a relative mean amplitude of $\left\langle\left|\mathbf{A}_{\mathbf{s}}(\mathrm{L})\right|>|<| \mathbf{A}_{\mathbf{p}}(\mathrm{L})\right|>=0.1308$ for the signal and $<\left|\mathbf{A}_{\mathbf{i}}(0)\right|>/<$ $\left|\mathbf{A}_{\mathbf{p}}(\mathrm{L})\right|>=0.07964$ for the idler. Let us stress the remarkable gain of coherence of the counter-propagating idler wave 
with respect to the pump, which exceeds two orders of magnitude, $\Delta v_{i} / \Delta v_{p} \simeq 1 / 245$, as illustrated in Figs.5(a)-(c). We have always obtained an excellent transfer of incoherence from the pump to the signal, thus allowing the counter-propagating idler wave to evolve toward a highly coherent state $\left(\Delta v_{i} / \Delta v_{p} \simeq 10^{-2}-10^{-3}\right)$. This shows that the convection-induced phase-locking mechanism is efficient for both deterministic phase modulations and random phase fluctuations.
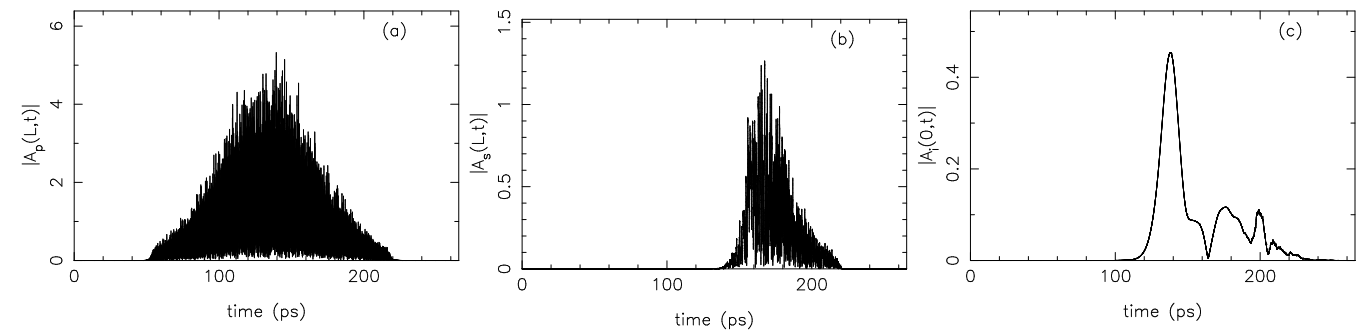

Fig. 4: Temporal amplitude evolution in the MOPO for (a) the forward pump pulse, (b) the forward signal, and (c) the backward idler (c).
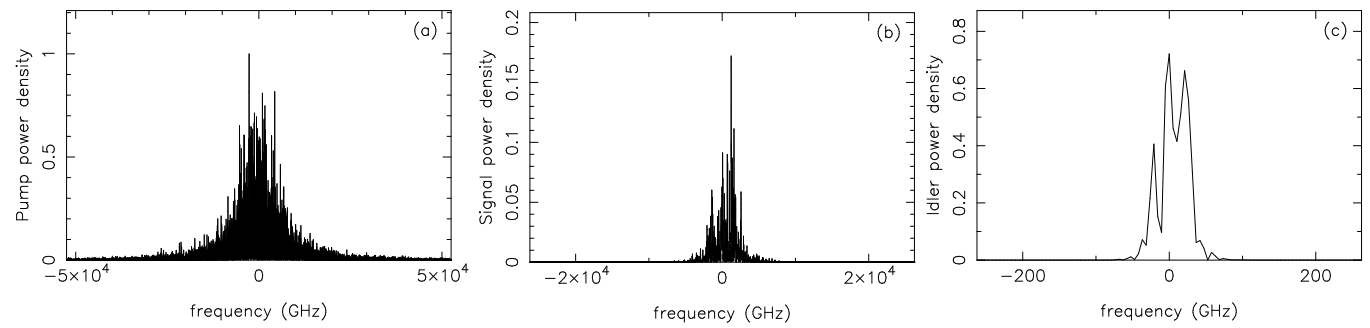

Fig. 5: (a) Incoherent pump spectrum of broad-bandwidth $\Delta v_{p}=15 \mathrm{Thz}$, (b) signal spectrum of $\Delta v_{s}=5.7$ Thz absorbing the pump stochasticity, and (c) backward idler spectrum with $\Delta v_{i}=57 \mathrm{Ghz}$ showing a coherence transfer of more than 2 orders of magnitude. [FSR $\left.=12 \mathrm{GHz} ; \Lambda_{0}=\mathrm{L} / 14=0.46 \mathrm{~mm} ; \tau_{0}=v_{p} / \Lambda_{0}=2.95 \mathrm{ps}\right]$

\subsection{Invariants conservation}

Let us make a brief comment concerning the conservation of the number of photons in the three-wave mixing process. Neglecting the dissipation $\left(\gamma_{j}=0, j=p, s, i\right)$, the total number of photons contained in the initial pump pulse namely,

$$
\mathrm{N}_{\mathrm{ph}}=\int_{-\infty}^{\infty}\left|\mathbf{A}_{\mathbf{p}}(\mathrm{x}, \mathrm{t}=0)\right|^{2} \mathrm{dx}
$$

must be conserved through the interaction in the crystal. More precisely, the balance equation for the output of the forward pump and signal waves at $\mathrm{x}=\mathrm{L}$ and of the idler at $\mathrm{x}=0$, should be written in the following form

$$
\int_{t=0}^{t=\infty}\left|\mathbf{A}_{\mathbf{p}}(\mathrm{x}=0, \mathrm{t})\right|^{2} \mathrm{dt}=\int_{t=0}^{t=\infty}\left|\mathbf{A}_{\mathbf{p}}(\mathrm{x}=\mathrm{L}, \mathrm{t})\right|^{2} \mathrm{dt}+\int_{t=0}^{t=\infty} \frac{\left|\mathbf{A}_{\mathbf{s}}(\mathrm{x}=\mathrm{L}, \mathrm{t})\right|^{2}}{\left(2 \sigma_{s} / \sigma_{p}\right)} \mathrm{dt}+\int_{t=0}^{t=\infty} \frac{\left|\mathbf{A}_{\mathbf{i}}(\mathrm{x}=0, \mathrm{t})\right|^{2}}{\left(2 \sigma_{i} / \sigma_{p}\right)} \mathrm{dt} .
$$

The numerical simulations conserve the total number of photons better than $10^{-5}$ for more than $6 \times 10^{6}$ time steps and high pump incoherence.

The dispersion relations of the backward three-wave interaction (TWI) process read

$$
\omega_{j}(k)=\mathrm{v}_{\mathrm{j}}+\frac{1}{2} \tilde{\beta}_{j} k^{2}\left(j=\underset{6}{p, s)} ; \quad \omega_{i}(k)=-\mathrm{v}_{\mathrm{i}}+\frac{1}{2} \tilde{\beta}_{i} k^{2}\right.
$$


where $\tilde{\beta}_{j}=2 \beta_{j} \mathrm{v}_{\mathrm{j}}^{2}$ and retaining only the first terms for the perturbative dispersion terms in Eqs.(2) they read

$$
\begin{aligned}
\left(\partial_{t}+\mathrm{v}_{\mathrm{p}} \partial_{x}+\gamma_{p}+i \frac{1}{2} \tilde{\beta}_{p} \partial_{x x}\right) \mathbf{A}_{\mathbf{p}} & =-\sigma_{p} \mathbf{A}_{\mathbf{s}} \mathbf{A}_{\mathbf{i}} \\
\left(\partial_{t}+\mathrm{v}_{\mathrm{s}} \partial_{x}+\gamma_{s}+i \frac{1}{2} \tilde{\beta}_{s} \partial_{x x}\right) \mathbf{A}_{\mathbf{s}} & =\sigma_{s} \mathbf{A}_{\mathbf{p}} \mathbf{A}_{\mathbf{i}}^{*} \\
\left(\partial_{t}-\mathrm{v}_{\mathrm{i}} \partial_{x}+\gamma_{p}+i \frac{1}{2} \tilde{\beta}_{i} \partial_{x x}\right) \mathbf{A}_{\mathbf{i}} & =\sigma_{i} \mathbf{A}_{\mathbf{p}} \mathbf{A}_{\mathbf{s}}^{*}
\end{aligned}
$$

These equations conserve the Manley-Rowe invariants

$$
\mathrm{I}_{1}=\frac{\mathrm{N}_{\mathrm{p}}}{\sigma_{p}}+\frac{\mathrm{N}_{\mathrm{s}}}{\sigma_{s}} ; \quad \mathrm{I}_{1}=\frac{\mathrm{N}_{\mathrm{p}}}{\sigma_{p}}+\frac{\mathrm{N}_{\mathrm{i}}}{\sigma_{i}}
$$

where $N_{j}=\int\left|A_{j}(x, t)\right|^{2} d x \quad(j=p, s, i)$. These invariants are also conserved in our simulations better than $10^{-5}$.

\section{3rd order MOPO-3}

According to the velocity-locking mechanism, the process of coherence transfer becomes more efficient when the group velocity of the co-propagating waves are matched [8], but this requires another choice of resonant frequencies for the backward MOPO [27]. In order to take advantage of a type I configuration in PPKTP, it is more convenient to chose the signal wave as the backward field, so that the group velocities of the co-propagating pump and idler waves may be matched exactly, $v_{p}=v_{i}$. Indeed, such a group velocity matching may be obtained for the following choice of the three wavelengths: $\lambda_{\mathrm{p}}=1060 \mathrm{~nm} ; \lambda_{\mathrm{s}}=1534 \mathrm{~nm}$; and $\lambda_{\mathrm{i}}=3429 \mathrm{~nm}$. The corresponding refractive indexes, group velocities and dispersion coefficients are: $\mathrm{n}_{\mathrm{p}}=1.8300, \mathrm{v}_{\mathrm{p}} / \mathrm{c}=0.5338, \beta_{\mathrm{p}} / \mathrm{v}_{\mathrm{p}}=0.1826 \mathrm{ps}^{2} / \mathrm{m} ; \mathrm{n}_{\mathrm{s}}=1.8163, \mathrm{v}_{\mathrm{s}} / \mathrm{c}=0.5401, \beta_{\mathrm{s}} / \mathrm{v}_{\mathrm{s}}=0.0761 \mathrm{ps}^{2} / \mathrm{m}$; $\mathrm{n}_{\mathrm{i}}=1.7709, \mathrm{v}_{\mathrm{i}} / \mathrm{c}=0.5338, \beta_{\mathrm{i}} / \mathrm{v}_{\mathrm{i}}=-0.5792 \mathrm{ps}^{2} / \mathrm{m}$. This point of operation is plotted on the group velocity dispersion curves reported in figure 6 .

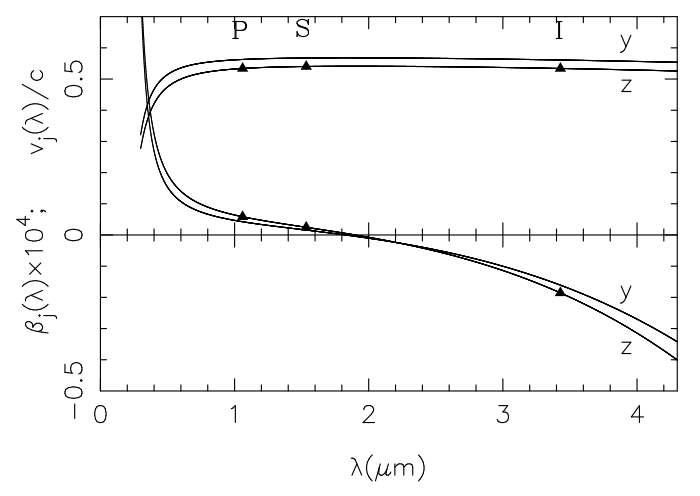

Fig. 6: Group velocity dispersion for $\mathrm{KTiOPO}_{4}$ at $20{ }^{\circ} \mathrm{C}$ (MOPO-3 of type I).

Let us note however that this configuration has a major drawback. The modulation period of the nonlinear coefficient for the first order resonant grating should be as short as $417 \mathrm{~nm}$. In the following, we thus propose to consider the next odd order $m=3$ [11], which thus makes the polarization poled grating of $\Lambda_{\mathrm{QPM}}=1251 \mathrm{~nm}$ experimentally accessible. For a $1 \mathrm{~cm}$ PPKTP crystal lenght, the oscillation threshold would be now $I_{\mathrm{pth}}(m=3)=9 I_{\mathrm{pth}}(m=1)=4.95 \mathrm{GW} / \mathrm{cm}^{2}$, which would be lower than the damage threshold for a $100 \mathrm{ps}$ pump pulse. We performed numerical simulations of this configuration by considering an input pump intensity of $I_{\mathrm{p}}=5 \mathrm{GW} / \mathrm{cm}^{2}$. In Eqs.(2) we must change the sign of the signal and the idler group velocities, because the counter-propagating wave now refers to the signal component, and $\Lambda_{\mathrm{QPM}}=\left[n_{p} / \lambda_{p}+n_{s} / \lambda_{s}-n_{i} / \lambda_{i}\right]^{-1}$ is the new modulation period of the quadratic nonlinear coefficient. Now, this configuration, perfectly satisfies the phaselocking condition (7) where the idler takes the place of the signal, namely $\left|v_{i}-v_{p}\right| \ll \lambda_{c} \gamma_{i}=v_{p} \tau_{c} \gamma_{i}$.

The results of the numerical simulations are reported in figures 7 and 8 . We consider here a highly incoherent pump pulse 
of $35 \mathrm{THz}$ bandwidth and $100 \mathrm{ps}$ duration. As expected from the convection-induced phase-locking mechanism, almost all the incoherence of the pump wave is transferred to the co-propagating idler wave, which thus leads to the generation of a highly coherent backward signal pulse. As remarkably shown in the figure 8(a)-(c), the gain of coherence of the signal with respect to the pump now exceeds three orders of magnitude.
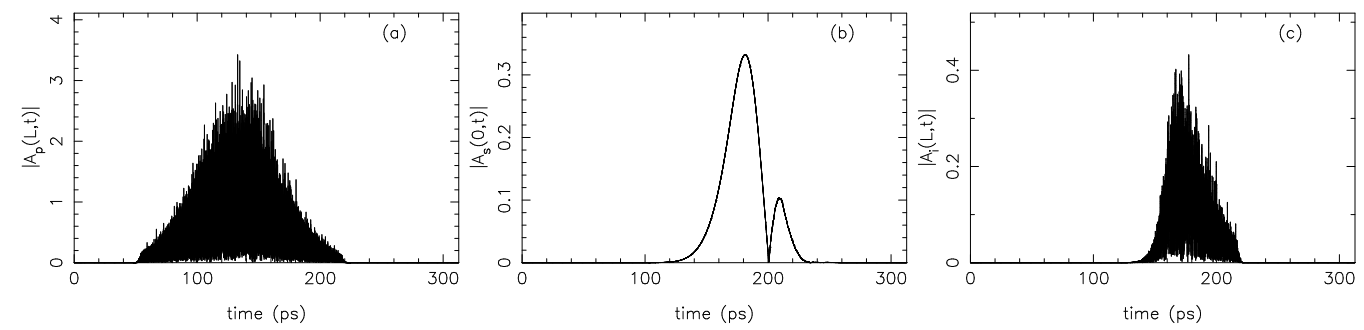

Fig. 7: Temporal amplitude evolution in the MOPO3 for (a) the forward pump pulse, (b) the backward signal, and (c) the forward idler (c).
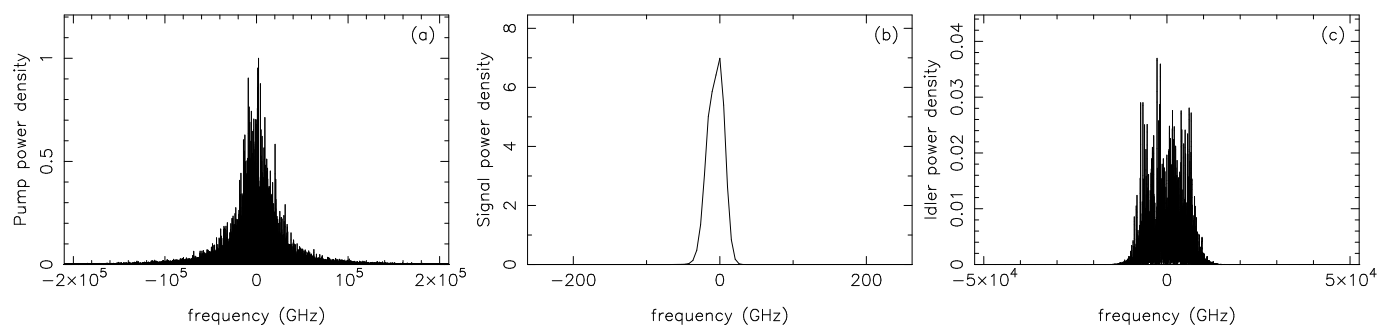

Fig. 8: (a) Incoherent pump spectrum of broad-bandwidth $\Delta v_{p}=35 \mathrm{Thz}$, (b) backward coherent signal spectrum of $\Delta v_{s}=28$ Ghz showing a coherence transfer of more than 3 orders of magnitude, and (c) forward idler spectrum with $\Delta v_{i}=17 \mathrm{Thz}$ absorbing the pump stochasticity. [FSR $=12 \mathrm{GHz} ; \Lambda_{0}=L / 14=0.46 \mathrm{~mm} ; \tau_{0}=v_{p} / \Lambda_{0}=2.95 \mathrm{ps}$ ]

\section{Conclusion}

In summary, we have shown that singly backward MOPOs pumped by an incoherent field are characterized, as a general rule, by the generation of a highly coherent backward wave. This remarkable property finds its origin in the convectioninduced phase-locking mechanism, in which the incoherence of the pump is absorbed by the co-propagating wave, thus allowing the backward wave to evolve toward a highly coherent state [7]-[10]. On the basis of the recent experiments [2] [6], we propose other realistic experimental conditions that may be implemented with currently available technology and in which backward coherent wave generation from incoherent excitation may be observed and studied. We note that it would be interesting to analyze theoretically these phenomena by making use of the kinetic wave theory [28]-[30], in line with the recent works on optical wave thermalization [31]-[37] and condensation [38]-[42]. Furthermore, a detailed analysis of the dynamics of the MOPO may reveal some connection with a class of solitary wave solutions spontaneously generated in the backward configuration of the three-wave interaction [19] [20].

\section{Acknowledgements}

The authors thank G. Strömqvist and V. Pasiskevicius for stimutating discussions and the GDR PhoNoMi2 $\mathrm{n}^{\mathrm{o}} 3073$ of the CNRS (Centre National de la Recherche Scientifique) devoted to Nonlinear Photonics in Microstructured Materials. 


\section{References}

[1] Harris S.E., "Proposed backward wave oscillation in the infrared", Appl. Phys. Lett. 9, 114-116 (1966).

[2] Canalias C. and Pasiskevicius V., Nature Photon. "Mirrorless optical parametric oscillator", 1, 459-462 (2007).

[3] Pasiskevicius V., Canalias C., Strömqvist G., and Laurell F., "Mirrorless OPO: first steps towards unlocking the potential of counter-propagating three-wave interactions" (invited), SPIE Proc. 6875, 6875081-12 (2008).

[4] Strömquist G., Pasiskevicius V., Canalias C. and Montes C., "Transform-Limited Mid-Infrared Pulses by Mirrorless Optical Parametric Oscillator", Middle Infrared Coherent Sources MICS’2009, Trouville, 8-12 June, 2009.

[5] Strömquist G., Pasiskevicius V., Canalias C. and Montes C., "Middle Infrared Coherent Source in Mirrorless Optical Parametric Oscillator", CLEO-EQEC, Munich, 15-19 June, 2009.

[6] Strömquist G., Pasiskevicius V., Canalias C. and Montes C., "Coherent phase-modulation transfer in counterpropagating parametric down-conversion", Phys. Rev. A 84, 023825-1-4 (2011).

[7] Picozzi A. and Hælterman M., "Parametric Three-Wave Soliton Generated from Incoherent Light", Phys. Rev. Lett. 86, 2010 (2001).

[8] Picozzi A., Montes C., and Hælterman M., "Coherence properties of the parametric three-wave interaction driven from an incoherent pump", Phys. Rev. E 66, 056605-1-14 (2002).

[9] Montes C., Picozzi A., and Gallo K., Opt. Commun. "Ultra-coherent output from an incoherent cw-pumped singly resonant optical parametric oscillator", 237, 437-449 (2004).

[10] Montes C., Grundkötter W., Suche H., and Sohler W., "Coherent signal from incoherently cw-pumped singly resonant Ti:LiNbO3 integrated optical parametric oscillators", J. Opt. Soc. Am. B 24, 2796-2806 (2007).

[11] Ding Y.I. and Khurgin J.B., "Backward optical parametric oscillators and amplifiers", IEEE J. Quantum Electron. 22, 1574-1562 (1996).

[12] Armstrong J.A., Bloembergen N., Ducuing J. and Pershan P.S., "Interaction between light waves in nonlinear dielectric", Phys. Rev., 127, 1918-1939, (1962).

[13] Sohler W. and Suche H., in Digest of the Third International Conference on Integrated Optics and Optical Fiber Communication (Optical Society of America, 1981), p.89

[14] Suche H. and Sohler W., "Integrated optical parametric oscillators" (invited), Optoelectronics-Devices and Technologies, Japan, 4(1) p.1-20 (1989).

[15] Arbore M.A. and Fejer M.M., "Singly resonant optical parametric oscillation in periodically poled lithium niobate waveguides", Opt. Lett. 22, 151-153 (1997).

[16] Hofmann D., Herrmann H., Schreiber G., Grundkötter W., Ricken R., and Sohler W., "Continuous-wave mid-infrared optical parametric oscillators with periodically poled $\mathrm{Ti}: \mathrm{LiNbO}_{3}$ waveguide", in 9th European Conference on Integrated Optics and Technical Exhibition ECIO'99 (EOS - European Optical Society 1999), p. 21.

[17] Hofmann D., Herrmann H., Schreiber G., Haase C., Grundkötter W., Ricken R., and Sohler W., in Nonlinear Guided Waves and Their Applications (Optical Society of America, 1999), FC2-1-3, p.465.

[18] Picozzi A. and Aschieri P., "Influence of dispersion on the resonant interaction between three incoherent waves", Phys. Rev. E 72, 046606-1-11 (2005).

[19] Montes C., Mikhailov A., Picozzi A., and Ginovart F., "Dissipative three-wave structures in stimulated backscattering. I. A subluminous solitary attractor", Phys. Rev. E, 55, 1086-1091 (1997).

[20] Montes C., Picozzi A., and Bahloul D., "Dissipative three-wave structures in stimulated backscattering. II. Superluminous and subluminous solitons", Phys. Rev. E, 55, 1091-1106 (1997). 
[21] Baltuska A., Berzanskis A., Gadonas R., Pugzlys A., Smilgevicius V., and Stabinis A., "Angular structure formation in single-pass optical parametric generators pumped by intersecting beams", Opt. Lett. 21, 2174 (1995)

[22] Marcinkevicius A., Piskarskas A., Smilgevicius V., and Stabinis A., "Parametric superfluorescence excited in a nonlinear crystal by two uncorrelated pump beams", Opt. Comm. 158, 101 (1998).

[23] Dubietis A., Danielius R., Tamosauskas G., and Piskarskas A., "Combining effect in a multiple-beam-pumped optical parametric amplifier", J. Opt. Soc. Am. B 15, 1135 (1998).

[24] Piskarskas A., Smilgevicius V., and Stabinis A., "Optical parametric oscillation excited by an incoherent conical beam", Opt. Comm. 143, 72-74 (1997).

[25] Piskarskas A., Smilgevicius V., Stabinis A. and Vaicaitis V. "Spatially cumulative phenomena and output patterns in optical parametric oscillators and generators pumped by conical beams", J. Opt. Soc. Am. B 16, 1566 (1999).

[26] Yan Yan and Changxi Yang, "Coherent light wave generated from incoherent pump light in nonlinear Kerr medium", J. Opt. Soc. Am. B 26, 2059 (2009).

[27] Montes C., Picozzi A., Durniak C., and Taki M., "Backward three-wave optical solitons", Eur. Phys. J. Special Topics 173, 167-191 (2009).

[28] Zakharov V.E., L’vov V.S. and Falkovich G., Kolmogorov Spectra of Turbulence I (Springer, Berlin, 1992).

[29] Newell A.C., Nazarenko S., and Biven L., "Wave turbulence and intermittency", Physica D 152-153, 520-550 (2001).

[30] Zakharov V.E., Dias F., and A. Pushkarev A., "One-dimensional wave turbulence", Phys. Rep. 398, 1 (2004).

[31] Pitois S., Lagrange S., Jauslin H.R., and Picozzi A., "Velocity Locking of Incoherent Nonlinear Wave Packets", Phys. Rev. Lett. 97, 033902 (2006).

[32] Lagrange S., Jauslin H.R., and Picozzi A., "Thermalization of the dispersive three-wave interaction", Europhys. Lett. 79, 64001 (2007).

[33] Picozzi A., "Spontaneous polarization induced by natural thermalization of incoherent light", Opt. Express 16, 17171 (2008).

[34] Barviau B., Kibler B., Coen S., and Picozzi A., "Toward a thermodynamic description of supercontinuum generation", Opt. Lett. 33, 2833 (2008).

[35] Picozzi A., Pitois S., and Millot G., "Spectral Incoherent Solitons: A Localized Soliton Behavior in the Frequency Domain", Phys. Rev. Lett. 101, 093901 (2008).

[36] Bortolozzo U., Laurie J., Nazarenko S., and Residori S., "Optical wave turbulence and the condensation of light", J. Opt. Soc. Am. B 26, 2280 (2009).

[37] Barviau B., Kibler B., Kudlinski A., Mussot A., Millot G., and Picozzi A., "Experimental signature of optical wave thermalization through supercontinuum generation in photonic crystal fiber", Opt. Express 17, 7392 (2009).

[38] Dyachenko S., Newell A.C., Pushkarev P., and Zakharov V.E., "Optical turbulence: weak turbulence, condensates and collapsing filaments in the nonlinear Schrödinger equation", Physica D 57, (1992) 96.

[39] Connaughton C., Josserand C., Picozzi A., Pomeau Y, and Rica S., "Condensation of Classical Nonlinear Waves", Phys. Rev. Lett. 95, 263901 (2005).

[40] Zakharov V.E. and Nazarenko S.V., "Dynamics of the Bose-Einstein condensation", Physica D 201, 203 (2005).

[41] Picozzi A., "Towards a nonequilibrium thermodynamic description of incoherent nonlinear optics", Opt. Express 15, 9063 (2007).

[42] Picozzi A. and Rica S., "Coherence absorption and condensation induced by thermalization of incoherent fields", Europhys. Lett. 84, 34004 (2008). 